\title{
Mechanical deformation of atomic-scale metallic contacts: Structure and mechanisms
}

Sørensen, Mads Reinholdt; Brandbyge, Mads; Jacobsen, Karsten Wedel

Published in:

Physical Review B

Link to article, DOI:

10.1103/PhysRevB.57.3283

Publication date:

1998

Document Version

Publisher's PDF, also known as Version of record

Link back to DTU Orbit

Citation (APA):

Sørensen, M. R., Brandbyge, M., \& Jacobsen, K. W. (1998). Mechanical deformation of atomic-scale metallic contacts: Structure and mechanisms. Physical Review B, 57(6), 3283-3294.

https://doi.org/10.1103/PhysRevB.57.3283

\section{General rights}

Copyright and moral rights for the publications made accessible in the public portal are retained by the authors and/or other copyright owners and it is a condition of accessing publications that users recognise and abide by the legal requirements associated with these rights.

- Users may download and print one copy of any publication from the public portal for the purpose of private study or research.

- You may not further distribute the material or use it for any profit-making activity or commercial gain

- You may freely distribute the URL identifying the publication in the public portal 


\title{
Mechanical deformation of atomic-scale metallic contacts: Structure and mechanisms
}

\author{
Mads R. Sørensen, Mads Brandbyge, and Karsten W. Jacobsen \\ CAMP, Department of Physics, Technical University of Denmark, DK-2800 Lyngby, Denmark
}

(Received 27 May 1997)

\begin{abstract}
We have simulated the mechanical deformation of atomic-scale metallic contacts under tensile strain using molecular dynamics and effective medium theory potentials. The evolution of the structure of the contacts and the underlying deformation mechanisms are described along with the calculated electronic conductance. Various defects such as intersecting stacking faults, local disorder, and vacancies are created during the deformation. Disordered regions act as weak spots that reduce the strength of the contacts. The disorder tends to anneal out again during the subsequent atomic rearrangements, but vacancies can be permanently present. The transition states and energies for slip mechanisms have been determined using the nudged elastic band method, and we find a size-dependent crossover from a dislocation-mediated slip to a homogeneous slip when the contact diameter becomes less than a few $\mathrm{nm}$. We show that the results measured in a nanocontact experiment depend significantly on the elastic stiffness of the experimental apparatus. For a soft setup, some of the atomic rearrangements might not be detected, whereas others are amplified. [S0163-1829(98)01306-X]
\end{abstract}

\section{INTRODUCTION}

The mechanical behavior of nanosize metallic contacts is of fundamental interest, for instance, in the field of tribology. Since macroscopic surfaces usually are rough, a contact between two macroscopic bodies consists of a large number of microscopic junctions. The process of moving two contacting surfaces with respect to each other involves the creation, deformation, and fracture of such junctions. Their mechanical properties are therefore important for phenomena such as adhesion, friction, and wear. Another interesting aspect of metallic point contacts originates from the electronic transport properties. As a contact between two metallic electrodes shrinks to atomic size, the diameter of the constriction becomes comparable to the Fermi wavelength in metals, and this gives rise to quantum features.

The opportunities for investigating single metallic contacts of nanometer size have been greatly improved by the inventions and refinements of experimental techniques such as the scanning tunneling microscope ${ }^{1}$ (STM) and the mechanically controllable break junction (MCBJ). ${ }^{2}$ In STM experiments, a tip-sample contact can be created by indenting a tip into a sample surface or by applying a voltage pulse between the tip and sample. The contact can then be manipulated by moving the tip with respect to the sample while monitoring the electronic conductance. ${ }^{3-13}$ With the MCBJ technique, a metallic wire is stretched and broken by bending a flexible substrate on which the wire is attached. Afterwards, the two electrodes can be brought repeatedly in and out of contact. ${ }^{2,14-16}$ Experiments have been performed under various conditions, such as ultrahigh-vacuum or ambient conditions, and at various temperatures, for instance, $4.2 \mathrm{~K}$ or room temperature. In the experiments, the indentationretraction process is highly irreversible. During tip-surface (electrode-electrode) separation, an atomic-size connective neck can be pulled. The conductance of such contacts exhibits sudden changes ("jumps") upon elongation or compression. More recently, the conductance and force have been measured using a STM supplemented by a force sensor, ${ }^{11-13}$ and in these experiments, a clear correlation between jumps in the force and jumps in the conductance was observed. The conductance of nanosize metallic contacts has also been studied with much simpler experimental methods. These studies include simple tabletop experiments with macroscopic metal wires, ${ }^{17-19}$ pin-plate experiments, ${ }^{20}$ and measurements on commercial or home-built electromechanical relays. ${ }^{21}$ These experiments demonstrate that even for contacts between macroscopic metallic bodies, the very last connective junction before separation is of atomic dimensions.

Early molecular dynamics (MD) simulations by Landman and co-workers predicted that when a metal tip is separated from a metal sample after an indentation, a connective neck can be pulled between the tip and sample, and during the tip retraction, the neck is elongated until fracture. ${ }^{22}$ In the simulations, the elongation of the neck consisted of a sequence of alternating elastic and plastic stages accompanied by atomic structural rearrangements. A similar irreversible loadingunloading behavior and neck formation was found in simulations by Sutton and co-workers. ${ }^{23}$ In later theoretical work, the STM and MCBJ experiments have been modeled using atomistic simulations combined with conductance calculations, which have been based on a tight-binding model, ${ }^{24-26}$ a free-electron model, ${ }^{6,9,25}$ or a semiclassical Sharvin expression. $^{20}$ The jumps in the conductance are generally associated with atomic rearrangements where the structure and cross-sectional area suddenly change, in agreement with recent combined force and conductance measurements. ${ }^{11-13}$ Simpler models of the evolution of atomic-scale metallic contacts have also been applied. ${ }^{27}$

In the present paper, we provide a detailed description of the evolution of the atomic structure of metallic nanocontacts and of the mechanical deformation processes that take place during an elongation process. In today's point contact experiments, one is restricted to measurements of a few physical quantities such as the conductance and force, and the challenge is to deduce as much information as possible about the properties of the contact. By showing how the structures and deformation mechanisms affect the measurable quantities in 


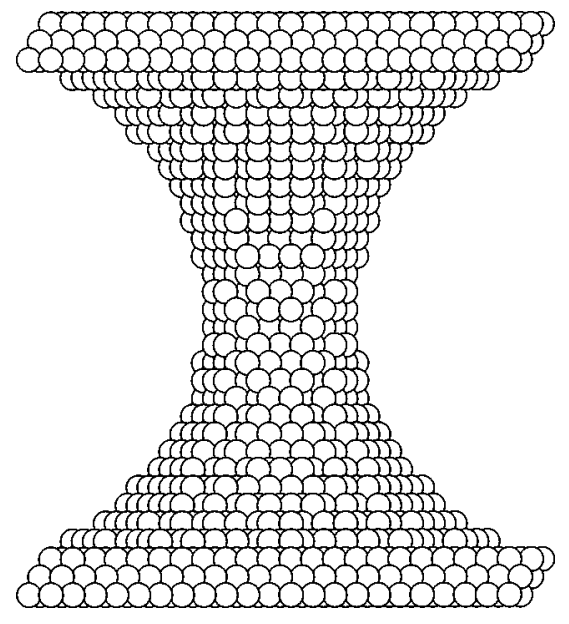

FIG. 1. A Au nanocontact oriented along the [111] direction. The contact has a length of 33 layers and consists of 6798 atoms. The diameter of the circular cross section at the thinnest point is $18.6 \AA$.

the simulations, our results should be useful in interpretations of experimental results. When comparing simulation results directly to experiments, one should be careful in case the experimental apparatus has a small stiffness. We have modeled the effect of a finite stiffness of an experimental setup, and it is shown that the results measured with a soft setup are significantly different from those obtained with a stiff setup.

\section{COMPUTATIONAL METHODS}

We have performed MD simulations of various metallic nanocontacts. The starting point is a neck that has a perfect fcc structure and a circular cross section with a diameter of approximately 6.5 times the distance between nearestneighbor atoms $\left(d_{\mathrm{NN}}\right)$, at the thinnest point. The shape of the contact is such that the neck diameter increases as a cosine function of $z-z_{\text {center }}$, where $z$ denotes the coordinate along the neck axis. The neck is attached to a slab at the top and at the bottom, and periodic boundary conditions are applied in all three dimensions. An example is shown in Fig. 1. We have performed simulations for two metals, Au and Ni; two temperatures, $12 \mathrm{~K}$ and $290 \mathrm{~K}$; and three crystal orientations of the neck axis, [111], [110], and [100].

Initially, the positions of the atoms are relaxed to a local minimum of the potential energy using a minimization technique similar to steepest descent, and after that, the system is equilibrated at the specified temperature for $100 \mathrm{ps}$. The stretching of the contact is simulated by constant temperature MD, where the length of the simulation box (in the $z$ direction) is increased at a constant rate. The temperature is kept fixed using a Langevin algorithm with fluctuating forces and frictional forces for all atoms in the system. ${ }^{28,29}$ A time step of $\Delta t=5.4 \times 10^{-15} \mathrm{~s}$ is used in the numerical integration of the equations of motion. ${ }^{30}$ The box length is increased by 1 $\times 10^{-4} \AA$ in each time step, corresponding to a velocity of 2 $\mathrm{m} / \mathrm{s}$. The interatomic forces and energies are calculated using semiempirical potentials derived from the effective medium theory (EMT).$^{31-33}$ In the EMT, the total energy of a system of atoms is written in the form

$$
E_{\mathrm{tot}}=\sum_{i}\left[E_{\mathrm{c}, i}\left(n_{i}\right)+\frac{1}{2} \sum_{j \neq i} V\left(r_{i j}\right)\right],
$$

where the sum is over all atoms in the system. The energy term $E_{\mathrm{c}, i}\left(n_{i}\right)$ for an atom is a function of an "embedding electron density," $n_{i}$, which is calculated as a superposition of density contributions from the surrounding atoms,

$$
n_{i}=\sum_{j \neq i} \Delta n_{j}\left(r_{i j}\right)
$$

Many-body effects, which are very important in metallic systems, are included in the description through the $E_{\mathrm{c}, i}$ term. The EMT potentials provide an approximate and computationally efficient description of the bonding in metallic systems, and they have been applied successfully in studies of, e.g., diffusion and surface relaxations and reconstructions. ${ }^{32}$

We do not find any important differences between the results obtained at $12 \mathrm{~K}$ and $290 \mathrm{~K}$, respectively, in our simulations, but this should not be taken as an indication that there is no temperature dependence in a nanocontact experiment, since there is a large difference in time scale between a MD simulation and an experiment. In a typical STM experiment, the tip is retracted by a velocity of $100 \AA / \mathrm{s}$, which is eight orders of magnitude slower than in our simulations. Thus, a process that occurs in an experiment at a given temperature might not take place in a MD simulation at the same temperature; for instance, diffusion processes might be neglected. Since the time scale in the simulations is short and the temperatures are far below the melting point, the results are not much different from those of a local minimization. The results presented in the figures below are from the simulations at $12 \mathrm{~K}$ where the thermal fluctuations are smallest.

\section{A. Common neighbor analysis}

The MD simulations produce a large amount of data. We obtain the positions and momenta of all the atoms in the system at each time step, and from the data, any piece of information about the evolution of the atomic structure is available, but it is not straightforward to communicate this information in a clear and concise manner. We have found it useful to analyze the structure of the contacts using the common neighbor analysis (CNA) method. ${ }^{34,35}$ In the CNA, a set of three integers, $j k l$, is assigned to each pair of neighbor atoms. A pair of neighbor atoms is also called a "bond." Two atoms are considered to be neighbors if they are within a certain distance, $r_{\text {cut }}$, of each other. The first index, $j$, is the number of atoms that are neighbors to both atoms in the pair; these are called the common neighbors. The second index, $k$, is the total number of bonds between the $j$ common neighbors. The third index, $l$, is the number of bonds in the longest continuous chain formed by the $k$ bonds between the common neighbors. We have used a fixed cutoff distance, $r_{\text {cut }}$, of $1.2 d_{\mathrm{NN}}$.

We have calculated CNA indices for a number of atomic configurations extracted from the MD simulations. For the perfect, initial contacts, there are only pairs of type 421, 311, 211, and 200 present; these pairs are characteristic of compact fcc clusters. In perfect fcc bulk, all pairs are of type 421. During the deformation process, some of the pairs are converted into other types of pairs, indicating the presence of 
defects. The most important ones are the 422 pairs, which are signatures of hcp ordering. In perfect hcp bulk, $50 \%$ of the pairs are of type 422 while the other 50\% are of type 421 . We also look at pairs that are of type 411, 433, 544, or 555. In the literature, the latter three types of pairs have been ascribed to icosahedral ordering (or distorted icosahedral ordering), ${ }^{34,36}$ but in the present context, we will merely consider them as indicators of a local environment that is different from fcc and hep ordering. ${ }^{37}$

\section{B. Conductance and area}

Based on the atomic configurations generated by the MD simulations, we calculate the electronic conductance of the $\mathrm{Au}$ contacts during the stretching process. To calculate the conductance, a one-electron potential is generated from the atomic coordinates in the following way. The electronic density is constructed as a sum of free-atom electron densities. The potential is then generated from the density using the local density approximation (LDA). We describe the macroscopic electrodes by a free-electron model, which is reasonable in the case of $\mathrm{Au}$, and join the contact and electrodes in a smooth manner. ${ }^{38}$ We neglect scattering from the ionic cores and cut off the generated potential at an energy of $-\left(E_{F}+W\right)$ relative to the vacuum level, where $E_{F}(5.5 \mathrm{eV})$ and $W(5.5 \mathrm{eV})$ are the Fermi energy and work function, respectively. ${ }^{39}$ This non-self-consistent potential gives a good description of the corrugation near the boundary, which is essential. The quantum transmission of electrons through the three-dimensional potential is calculated using a numerical exact, recursive multichannel method. The electronic wave functions are expanded in plane waves using a $(x y)$ supercell perpendicular to the contact with side lengths $L_{x}$ $=L_{y}=40 \AA$. A three-dimensional grid with grid size $0.8 \AA$ and 400 plane waves (channels) is employed. The conductance is then determined from the Landauer expression linking the total transmission for electrons at $E_{F}$ and the conductance, ${ }^{40,41}$

$$
G=G_{0} T_{\text {tot }}\left(E_{F}\right) \text {. }
$$

The quantum unit of conductance, $G_{0}$, is $2 e^{2} / h$, where $e$ is the electronic charge and $h$ is Planck's constant. For further details about the computational procedure, we refer to Ref. 42 and references therein.

We have also found it useful to monitor the smallest cross-sectional area of the contact (perpendicular to the contact axis). The area of a cross section is calculated by a procedure used by Bratkovsky et al. in Ref. 25. Each atom is represented by a sphere with a volume of the fcc bulk Wigner-Seitz cell (radius $r_{s}$ ). At each $z$ position of interest, a slice with a thickness of an interlayer distance is centered. The area is calculated as the total volume of the atomic spheres overlapping with the slice divided by the thickness of the slice.

\section{RESULTS OF MOLECULAR DYNAMICS SIMULATIONS}

In this section, we present the results of the simulations of the nanocontacts. We have simulated two different metals: $\mathrm{Au}$ and Ni. Au was an obvious choice since it has been used

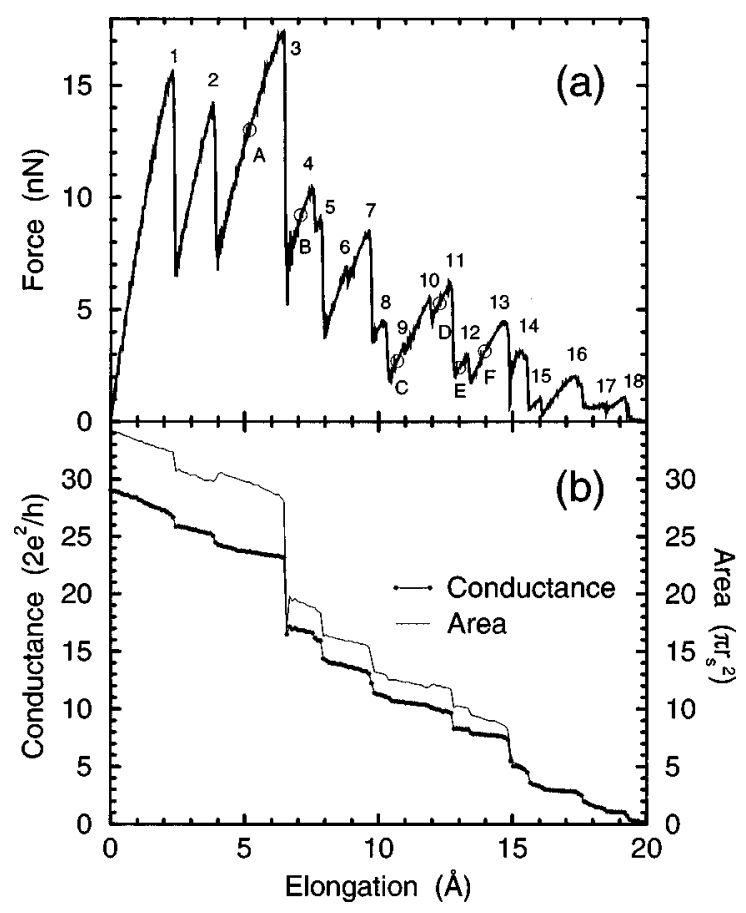

FIG. 2. Tensile force, $F$, conductance, $G$, and area, $A$, as a function of elongation for a $\mathrm{Au}[111]$ nanocontact. Each value of $F$ is an average over 1 ps corresponding to $0.02 \AA$, whereas a data point for $A$ and $G$ is calculated from a single atomic configuration for each $0.1 \AA$.

widely in experiments. Furthermore, the electronic structure of Au with a closed $d$ shell and a simple band structure justifies the use of a free-electron model for the conductance calculations. The choice of $\mathrm{Ni}$ as an alternative metal was based on the differences in quantities such as defect formation energies and elastic constants. For instance, the shear modulus and Young's modulus of $\mathrm{Ni}$ are much larger than those of Au. The simulation of the Au[111] contact is discussed thoroughly. First, we describe the gross features of the deformation of the contact with focus on measurable quantities such as the tensile force and electronic conductance. Second, we describe in detail how the atomic structure of the contact evolves, and we characterize the mechanical processes that take place. The $\mathrm{Au}[110]$ and $\mathrm{Au}[100]$ contacts are discussed more briefly with emphasis on features that are new compared to the $\mathrm{Au}[111]$ simulation. For each contact type (crystal orientation) we present and discuss the results of a single simulation, but the same general behavior has been observed in other similar simulations. Finally, the Ni results are compared to the Au results with respect to the strength, stiffness, and shape of the contacts.

\section{A. $\mathrm{Au}[111]$ contact}

In agreement with previous simulations, we find that the deformation of the contact in general proceeds by a sequence of atomic rearrangements separated by stages of elastic deformation. This behavior is reflected in the tensile force, conductance, and (smallest) cross sectional area, as shown in Fig. 2 for a $\mathrm{Au}[111]$ contact. In the elastic stages, the atomic structure remains basically unchanged while the contact is elongated. Meanwhile, the force increases smoothly, ap- 


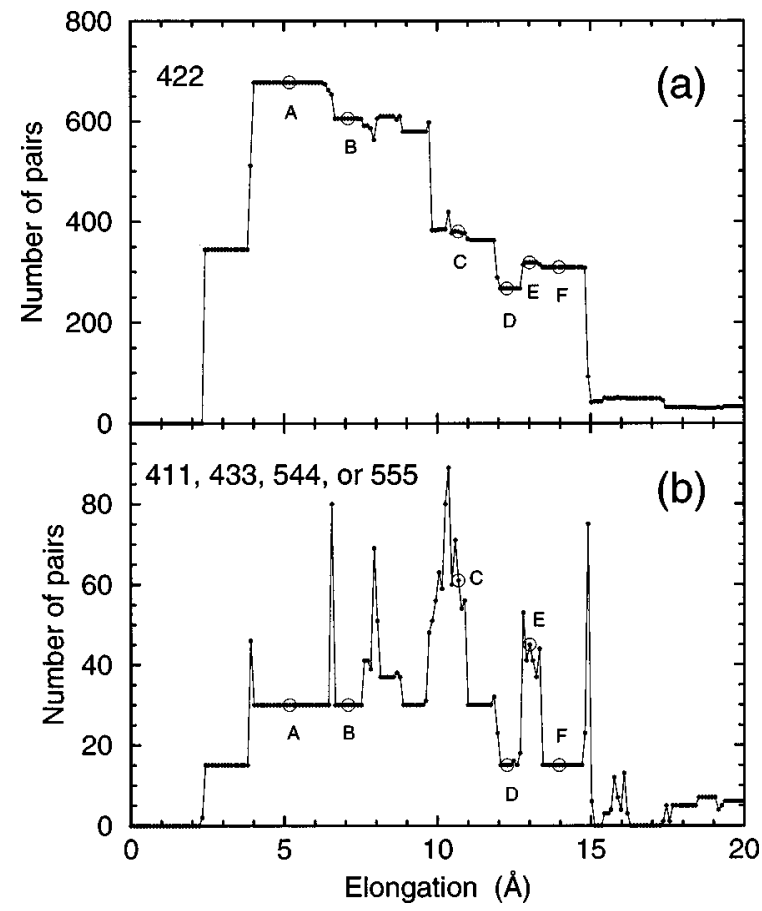

FIG. 3. CNA results for a $\mathrm{Au}[111]$ nanocontact. The number of various types of pairs are shown as a function of elongation. (a) The number of pairs of type 422. (b) The number of pairs of type 411, 433,544 , or 555 .

proximately linearly, and the conductance and area decrease smoothly. At certain points, when the tension in the wire has reached a high level, the system becomes mechanically unstable, and the atoms rearrange themselves into a different structure. A rearrangement almost always shows up as a sharp decrease (a jump) in the force [see labels 1-18 in Fig. 2(a)]. In most cases, a rearrangement also induces a sharper decrease of the conductance. There are, however, structural changes that do not affect the area at the thinnest point of the neck, or the conductance, much. For instance, the force jumps labeled $6,9,10$, or 15 do not have simultaneous, marked changes of conductance. We find that the force is a sensitive probe of changes in the atomic structure.

The separation (i.e., the elongation) between two successive force jumps is in general smaller than an interlayer distance. The elongation length between the 1st and the 17th jump is about $16.1 \AA$, which gives an average separation of $1.0 \AA$. This is less than half of the interlayer distance of 2.3 $\AA$. On the other hand, some force jumps are more pronounced than others. If one only considers the major jumps $(1-4,7,11,13-14,16)$, an average separation of $1.9 \AA$ is found. At the end of the elongation, just before the breakage, the contact has a single atom at the thinnest point, and the conductance is close to $G_{0}$. The pulloff force required to break the final one-atom contact is around $1.1 \mathrm{nN}$ in reasonable agreement with the value of $1.5 \pm 0.2 \mathrm{nN}$ reported by Rubio et al. ${ }^{13}$

Results for the common neighbor analysis of the $\mathrm{Au}[111]$ contact are shown in Fig. 3. There is a clear correlation between the number of CNA pairs in Fig. 3 and the force and conductance in Fig. 2. At the time of a force jump, the number of pairs of type 422 suddenly changes, indicating a structural change. In between the force jumps, the number of 422

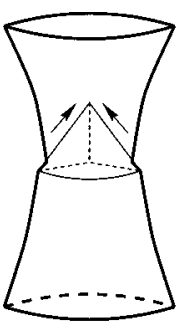

(a)

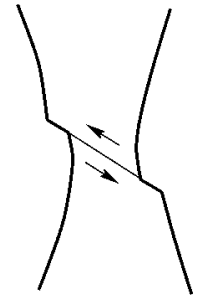

(b)

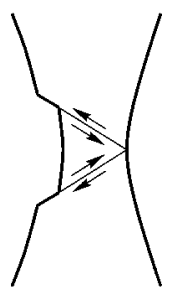

(c)
FIG. 4. Schematic illustration of selected slip processes. (a) A three-plane slip. (b) A simple single-plane slip. (c) A slip in two nonparallel slip planes.

pairs is fairly constant. The number of pairs of the types 411, 433,544 , or 555 behaves similarly, except that it fluctuates more in some stages. ${ }^{43}$

There are occasionally some "spikes" in the number of 411/433/544/555 pairs in Fig. 3(b). The spikes are associated with atomic rearrangements, and they could indicate that some intermediate, disordered structures are present. We have relaxed the extracted atomic configurations from the (low-temperature) MD simulations to a local potential energy minimum to make sure that we are dealing with structures that are at least metastable and not just configurations that were accidentally extracted at the time of one of the atomic rearrangements. We find that after the relaxations, some of the spikes have disappeared, while others remain, however reduced in amplitude. In other words, some of the configurations have turned into ordered structures similar to the structures that are present after the rearrangements, while others have ended up in disordered structures. The presence of the latter structures indicates that the mechanical rearrangements from one ordered structure to another might be incomplete and rather be composed of two rearrangements with a disordered structure in between. It is possible, however, that these intermediate structures might to some extent anneal out at higher temperatures and longer time scales.

In the 1st rearrangement, a partial slip has occurred in three nonparallel close-packed $\{111\}$ planes in a region above the center of the contact. In a fcc lattice there are four different sets of $\{111\}$ planes, which lie parallel to the sides of a regular tetrahedron. One set of planes, the (111) planes, are perpendicular to the $z$ axis. The other three sets of planes are inclined to the $z$ direction, and they are the active slip planes in which slip can occur to relieve the tensile stress in a contact during elongation. The three-plane slip is illustrated schematically in Fig. 4(a). The 2nd rearrangement is an almost exact mirror image of the first one, taking place in the lower part of the contact. At the time of the 1st rearrangement, the number of 422 pairs increases from zero to around 350 , and during the 2 nd rearrangement, it increases by almost the same amount to a total of nearly 700 . The number of 411/433/544/555 pairs increases from zero to 15 and 30 , respectively, and it is entirely an increase of 433 pairs. The increase in the number of 422 pairs is due to the formation of stacking faults caused by the slips in the three $\{111\}$ planes. Around an intrinsic stacking fault, the atoms in two $\{111\}$ layers find themselves in a hcp environment. A snapshot of the contact after the 2nd rearrangement is shown in Fig. 5(A). The atoms forming the non-fcc pairs are located in the 

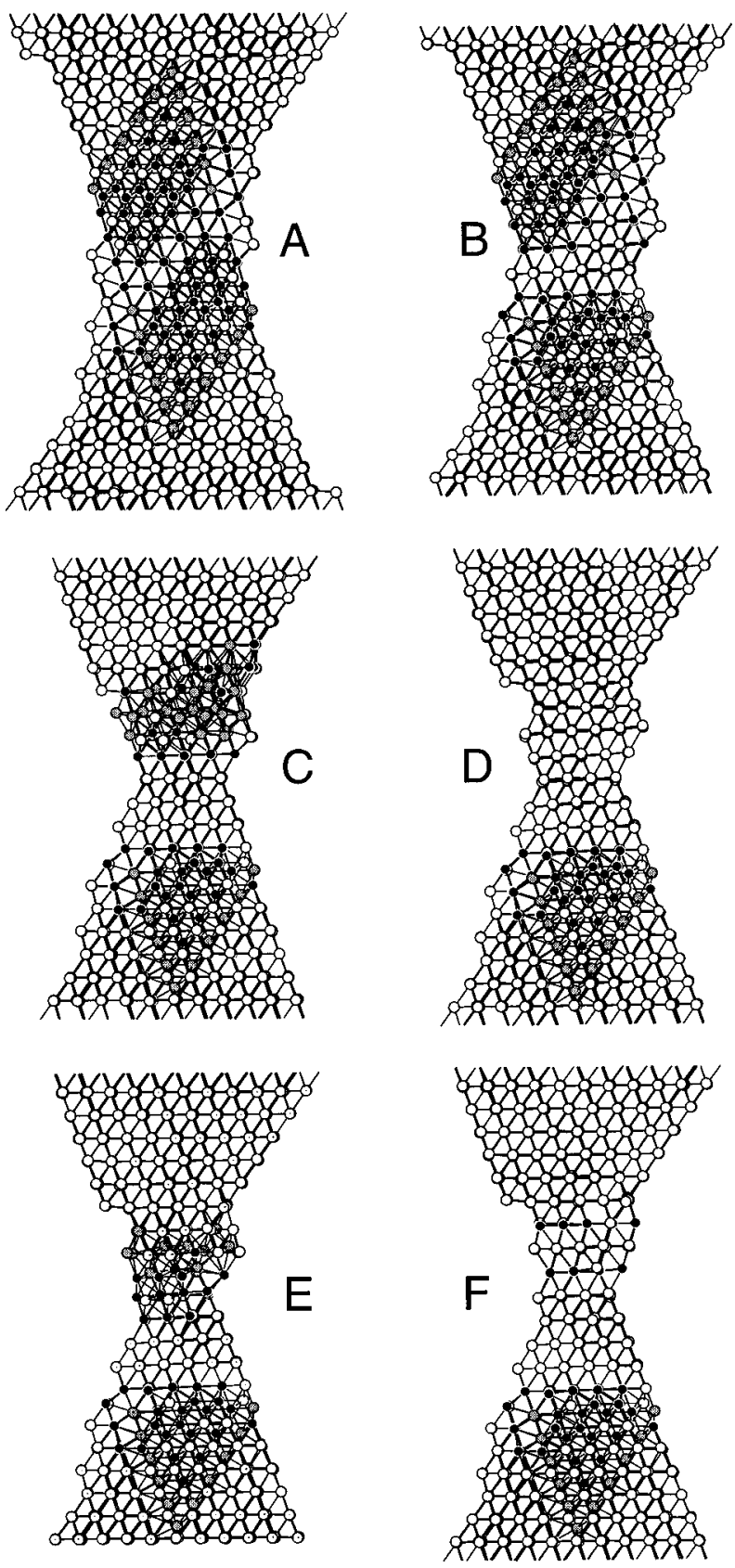

FIG. 5. Snapshots of atomic configurations of a $\mathrm{Au}[111]$ nanocontact. The atoms are colored according to their local environment as determined by CNA. Atoms that form at least one "bond" of type 411, 433, 544, or 555 are colored gray. Atoms that do not form any of these bonds but form at least one bond of type 422 are colored black. The remaining atoms are colored white. Only atoms in the "active" central region are shown.

two slip regions, one above and one below the center of the contact. The atoms forming 422 pairs are located around the slip planes (three sides of a tetrahedron), and the atoms forming 433 pairs are found at the intersection lines of the slip planes (three edges of a tetrahedron).

The structural defects created by the three-plane slip affect the electronic potential that determines the conductance. Inhomogeneities (scattering centers) are introduced in the potential at points where the local density of atoms is reduced [Fig. 6(A)]. The largest inhomogeneities are located at

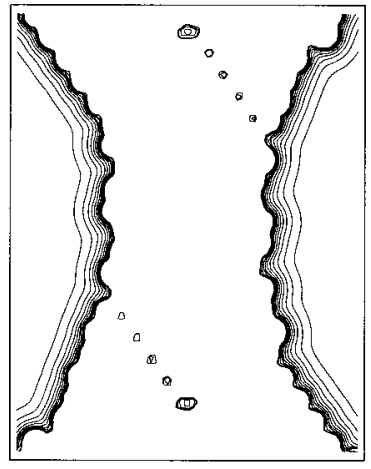

A

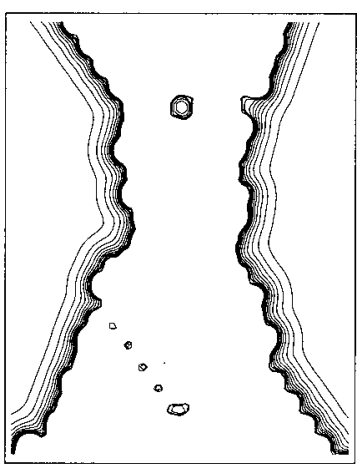

D
FIG. 6. Contours of the one-electron potential in a cross section through two configurations of a $\mathrm{Au}[111]$ contact. In configuration A, inhomogeneities (scattering centers) are present, in particular at the corners of the two slip-tetrahedra, and to a smaller extent at the edges of the tetrahedra, where two stacking faults meet. In configuration $\mathrm{D}$, a large inhomogeneity is present slightly above the center of the contact. This scattering center corresponds to a vacancy in an otherwise perfectly ordered region.

the corners of the "slip tetrahedra;" smaller ones are present at the edges of the tetrahedra.

We believe that the occurrence of a three-plane slip is a nanoscale effect driven by a reduction of the slip area. If a slip were to occur in a single plane for a [111] contact, the slip area would be very large, since the inclination angle between the possible slip planes and the $z$ direction is only $19.5^{\circ}$. Instead, the slip area is reduced by slipping in three planes, which form three sides of a tetrahedron inside the contact. For a wire of macroscopic dimensions, a dislocation can be regarded as a line defect and the size of the slip area is not important, but for nanocontacts, a slip is spread out over a significant part of the slip area and therefore it is favorable to reduce the area.

It has been common practice in the literature to discuss the structure and deformation of a nanocontact in terms of layers of atoms (perpendicular to the $z$ axis). The elongation has been described as happening in a layer-by-layer fashion. It is an intuitively appealing picture, but we find that it, in general, is somewhat oversimplified. For instance, for a three-plane slip, the atomic rearrangements extend over many layers in the contact, and as a result, a profile of the layer-by-layer structure would appear to be disordered in a large region of the contact. But disorder is not a proper description in this case since we are dealing with well defined defects, namely, stacking faults. We shall use the term "active zone" to refer to the region in the contact where the local atomic environment, given by the relative positions of the neighbor atoms, has been changed by the rearrangement.

Turning to the $3 \mathrm{rd}$ atomic rearrangement, a snapshot of a configuration after the rearrangement is shown in Fig. 5(B). The rearrangement is localized at the center of the contact. Some of the defects in the region are removed by the process; this is reflected as a decrease in the number of the 422 pairs in Fig. 3(a). Disregarding the irregular layer structure due to the stacking faults, it is reasonable to use the layerby-layer terminology and state that two layers of atoms are rearranged into three layers. This accounts for an unusually 
dramatic decrease in the cross-sectional area of approximately $1 / 3$ during the rearrangement.

The following seven rearrangements $(4-10)$ are difficult to characterize in simple terms. Slips play an important role in most cases, but they are accompanied by other atomic displacements, of which many are irregular compared to, e.g., the collective motion during a slip. The active zone extends in most cases over a large region in the upper part of the contact. The remarkable net result is that after the 10th rearrangement, the upper half of the contact appears to have a completely well ordered fcc structure [Fig. 5(D)]. There is, however, a vacancy present inside the contact and it clearly shows up in the one-electron potential used for the conductance calculations [Fig. 6(D)]. The effect of such a scattering source on the conductance has been investigated in Ref. 42. Along the way, there are stages in which the contact contains locally disordered structures. This is in particular the case between the 7th and 9th processes, and to a smaller extent between the 4th and 6th processes. The disorder shows up in a higher number of 411/433/544/555 pairs in Fig. 3(b). As an example, a snapshot of the system between the 8 th and the 9th rearrangements is seen in Fig. 5(C). The atomic rearrangements preferably take place at disordered regions, and the rearrangements tend to reduce the amount of disorder. The vacancy, which shows up in the potential in Fig. 6(D), is created during the 7th-9th rearrangements and it remains there for the rest of the elongation. At longer time scales and higher temperatures, vacancies might migrate to the surface by diffusion.

The 11th and the 12th rearrangements can be described as local order-disorder and disorder-order processes. In the 11th rearrangement, the ordering is destroyed in four (111) layers [Fig. 5(E)], and in the 12th process, the atoms in this active zone orders into five (111) layers [Fig. 5(F)]. The addition of an extra (111) layer gives rise to an extrinsic stacking fault, as visualized by the black atoms in Fig. 5(F).

The next rearrangement, the 13th, actually consists of two processes. In the middle of the contact, there is a local rearrangement where four (111) layers turn into five layers, but at the same time, the reverse of the 3rd rearrangement, i.e., the three-plane slip, occurs in the lower part of the contact. The rearrangements can be understood in the following way. The three-plane slip results in a rather unfavorable, strained structure, as mentioned above, and it takes a certain tensile force to keep this structure from "slipping back" to the perfect structure. As the contact gets thinner, the contact becomes unable of supporting a high tensile force. The 13th rearrangement begins in the middle of the contact where the local rearrangement induces a decrease in the tensile force to a low value [see the "dip" in the force in Fig. 2(a)]. At this point, the tensile force is too small to keep the unfavorable structure; the defected structure becomes unstable and disappears by a reverse three-plane slip.

The 14th and the 15th rearrangements are local orderdisorder and disorder-order processes, respectively, similar to the 11th and 12th rearrangements. After the 16th rearrangement and for the rest of the elongation, the structure at the narrowest point of the contact appears disordered. This is hardly surprising, considering the small thickness of the contact and the high state of stress. A snapshot of the system just before the final rupture is shown in Fig. 7(a).
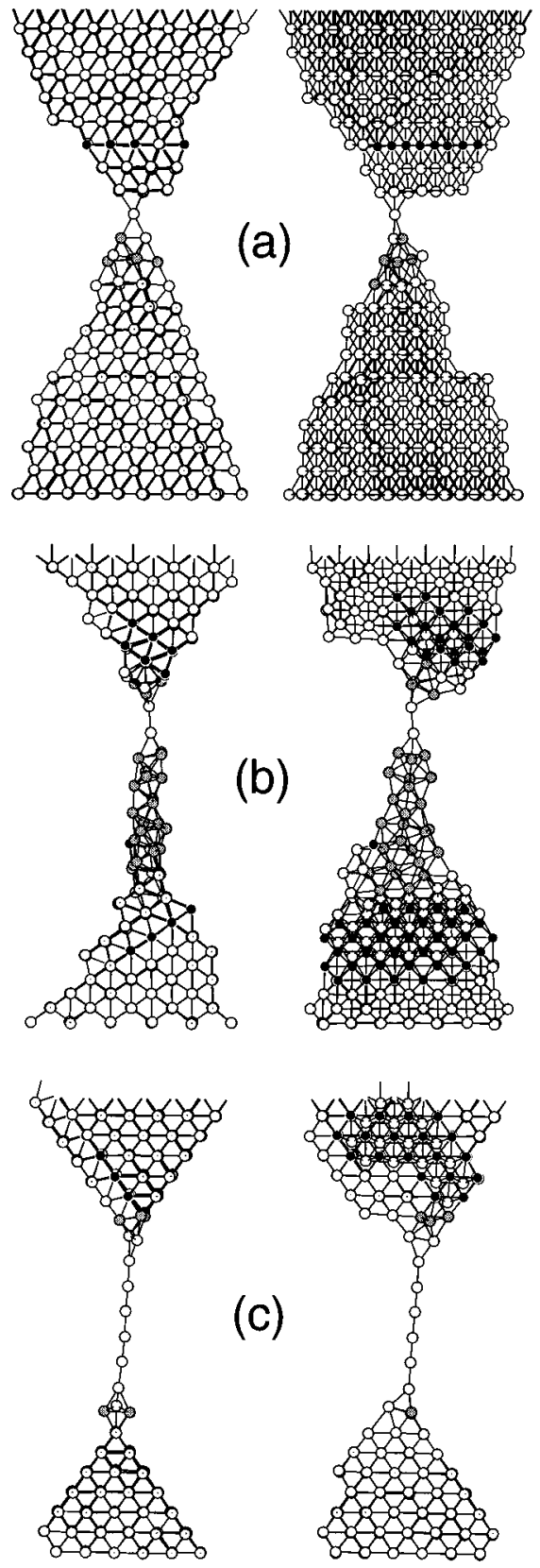

FIG. 7. Snapshots of atomic configurations from MD simulations of $\mathrm{Au}$ nanocontacts. The pictures show, from two different viewpoints, the final configuration just before rupture of three different contacts: (a) $\mathrm{Au}[111]$, (b) $\mathrm{Au}[110]$, and (c) $\mathrm{Au}[100]$.

There is a noteworthy correlation between the degree of ordering of the atomic structure and the strength of the nanocontact. The peak values of the tensile force just before the rearrangements tend to be smaller for disordered contacts (e.g., the 5th, 6th, 8th, 9th, and 12th rearrangements) compared to well-ordered contacts of similar thickness. The issue of the tensile strength of nanocontacts is discussed further in Sec. III D.

\section{B. Au[110] contact}

The deformation of the $\mathrm{Au}[110]$ contact differs substantially from that of the $\mathrm{Au}[111]$ contact. For a contact oriented along the [110] direction, two sets of close-packed $\{111\}$ 

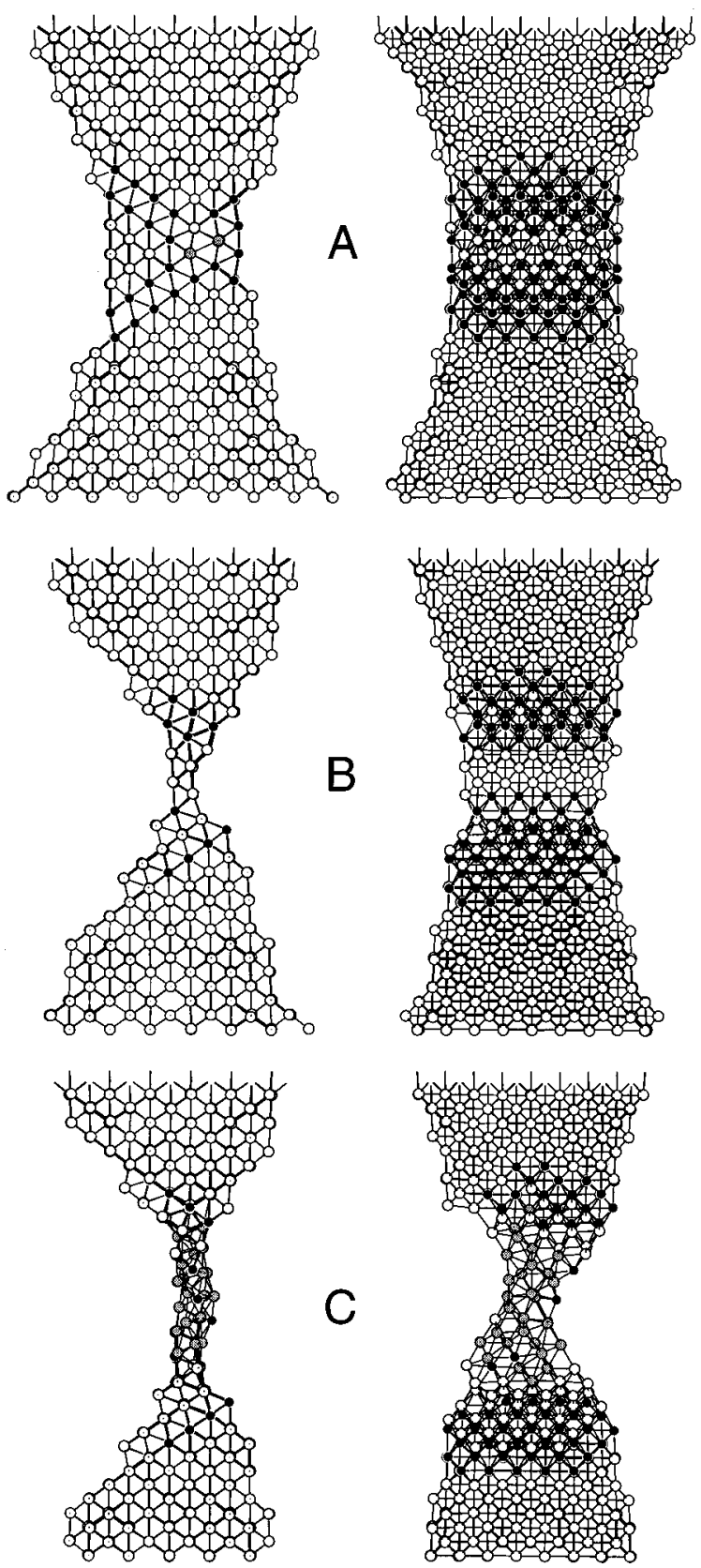

FIG. 8. Snapshots of atomic configurations of a $\mathrm{Au}[110]$ nanocontact from two different viewpoints. Left: Seen from the [1 $\overline{1} 0]$ direction. Right: Seen from the [001] direction.

planes lie parallel to the $z$ axis, and these planes are inactive with respect to slip. The remaining two sets of $\{111\}$ planes are the possible active slip planes. The inclination angle between the active slip planes and the $z$ direction is relatively large, $54.7^{\circ}$, and therefore, the slip area for a single-plane slip is relatively small for a [110] contact, as opposed to a [111] contact. The elongation can be divided into two stages.

In the first stage, which consists of the first 11 rearrangements, the deformation takes place by slip in the two active sets of slip planes. The 1 st and 2 nd rearrangements are ordinary partial slips, each occurring in a single $\{111\}$ slip plane. The slips leave behind two intersecting stacking faults [Fig. 8(A)]. Most of the slips are of this type, but others are more unusual. In the $3 \mathrm{rd}$, 4th, and 8th rearrangements, a cluster of atoms in the contact slips simultaneously in two nonparallel

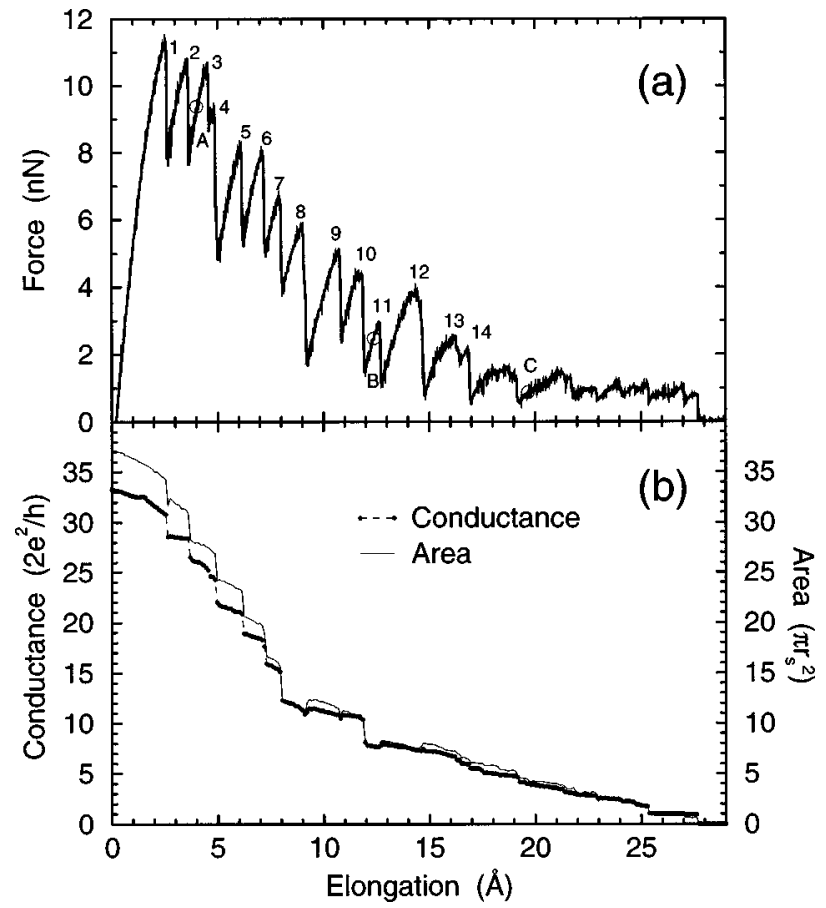

FIG. 9. Tensile force, conductance, and area as a function of elongation for $\mathrm{a} \mathrm{Au}[110]$ nanocontact.

slip planes at the stacking faults. The 1 st and 4 th processes are illustrated schematically in Figs. 4(b) and 4(c), respectively. The contact maintains a relatively ordered structure, apart from stacking faults and intersections between stacking faults. Due to the nature of the atomic rearrangements, the shape of the contact evolves asymmetrically. The cross section at the thinnest point becomes very oblong as opposed to the initial spherical shape [Fig. 8(B)].

When there are only two atoms in the thin direction of the smallest cross section, the nature of the deformation changes. In the second stage, the deformation consists of local, irregular rearrangements at the narrowest point of the contact. After the 14th rearrangement, the central region of the contact gets into a state of permanent disorder, which lasts until the contact breaks [Fig. 8(C) and Fig. 7(b)].

The difference between the two regimes is reflected in the force and conductance (Fig. 9) as well as the results of the CNA method (Fig. 10). In the slip regime, the force and conductance curves have a fairly regular sawtooth shape and staircase shape, respectively. The distances between the rearrangements are quite similar with an average of $1.0 \AA$; this is smaller than the interlayer distance of $1.4 \AA$. In the second regime, the shape of the force curve is more irregular, and the area decreases smoothly. The disorder shows up in the CNA results as an increase in the number of pairs of type $411,433,544$, or 555 [Fig. 10(b)]. The absolute number of these pairs is only around $20-50$, but it is significant since the pairs are located in the narrowest region of the contact.

\section{C. $\mathrm{Au}[100]$ contact}

For the $\mathrm{Au}[100]$ contact, the first six rearrangements are dominated by multiple slips in several different $\{111\}$ planes, but on top of that, there are other, more irregular atomic displacements. The active zone extends over 6-8 layers. 


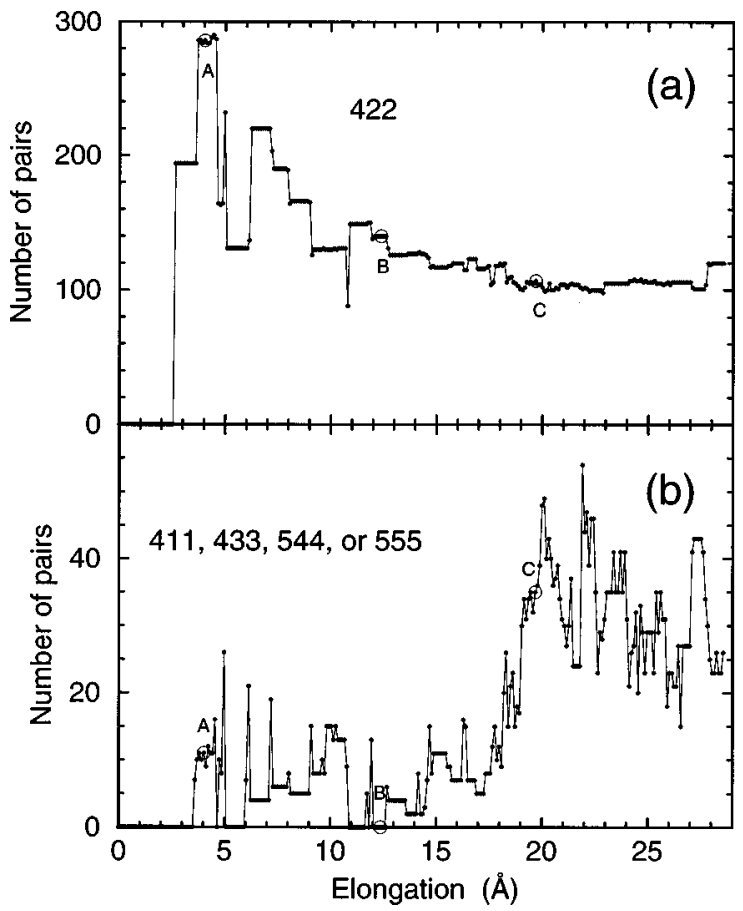

FIG. 10. CNA results for a $\mathrm{Au}[110]$ nanocontact.

During the elongation process, there are stages during which the contact is locally disordered. Although all four sets of $\{111\}$ planes can work as active slip planes for a [100]oriented contact, we find that slip mainly occurs in two sets of planes, and this leads to an oblong cross section of the contact, as for the $\mathrm{Au}[110]$ contact. The central region becomes permanently disordered in the last part of the simulation.

The most remarkable feature of the simulation is the formation of an unusually long chain of single atoms in the middle of the contact. Just before the final rupture, the chain consists of six atoms [Fig. 7(c)]. This is a rare structure in the simulations. As a result of this atomic structure, there is a plateau in the conductance curve at $G_{0}$ over an elongation length of about $10 \AA$. At this point we note that the interactions between the atoms in the simulation are described using an approximate method, and it is not clear how well the method works for the very low-coordinated atoms in the chain. Directional bonding effects might not be sufficiently accurately described. For comparison, we have determined the equilibrium interatomic distance, $d$, and force constant per atom, $K$, for an infinite, one-dimensional chain of $\mathrm{Au}$ atoms using density functional theory (DFT) calculations within the local density approximation with a plane wave basis set and pseudopotentials. ${ }^{44}$ For this worst-case example, the results of the DFT calculations are $d=2.54 \AA$ and $K=8.8 \mathrm{eV} / \AA^{2}$. The EMT potentials yield $d=2.47 \AA$ and $K$ $=15.0 \mathrm{eV} / \AA^{2}$. It is also possible that the long chain would not be stable at longer time scales and higher temperatures.

\section{Differences between Au and Ni}

The last part of the elongation process is particularly interesting in the context of conductance quantization. For $\mathrm{Au}$ contacts, we find a plateau in the conductance curve around $G_{0}$ when there is just a single atom in the thinnest cross section of the contact; however, the lengths of the plateaus are very different, as mentioned above. Steps at higher conductance values can also be seen (e.g., close to $3 G_{0}$ in Fig. 2 ), but scattering from the boundary and vacancies will typically downshift or smear out these plateaus somewhat, ${ }^{42}$ in accordance with experiments.

For $\mathrm{Ni}$, the electronic structure is more complicated due to the $d$ states and it is not reasonable to describe the electrodes by a free-electron model. Experiments show no statistical tendency for quantized conductance values in the case of Ni. ${ }^{19,45}$ Electronic effects might well be the reason why quantized conductance is not observed in $\mathrm{Ni}$ as opposed to Au. Returning to the mechanical behavior, we do find, however, that the mechanical behavior of $\mathrm{Ni}$ is different from that of $\mathrm{Au}$ in the final part of the elongation. Contacts of $\mathrm{Au}$ tend to be longer and smoother in the central region than in $\mathrm{Ni}$ contacts, and Au contacts can be stretched longer than $\mathrm{Ni}$ contacts (also when correcting for the difference in lattice constant). We often observe $\mathrm{Ni}$ contacts break at a cross section of two atoms, or more; this is seldom observed for Au contacts.

There are also some other quantitative differences between $\mathrm{Au}$ and $\mathrm{Ni}$ contacts, for instance, with respect to the strength and stiffness of the contacts. The tensile strength can be determined experimentally from measurements of the conductance and tensile force by dividing the force with the area estimated from the conductance. We find that the tensile strength of $\mathrm{Au}$ contacts is around 4-6 GPa, in good agreement with experimental measurements, ${ }^{11-13}$ whereas $\mathrm{Ni}$ contacts are significantly stronger with strengths in the range of 10-20 GPa. The elastic stiffness of the contacts depends on the kind of metal through the Young's modulus, which is higher for Ni than for Au by approximately a factor of 3 . Contacts of $\mathrm{Ni}$ are thus much stiffer than contacts of $\mathrm{Au}$.

\section{DISLOCATION GLIDE OR HOMOGENEOUS SLIP}

As described above, a slip between close-packed $\{111\}$ planes is an important mechanism for the deformation of the nanocontacts. A slip can take place by (at least) two distinct mechanisms: (1) a glide of a dislocation and (2) a homogeneous shear of one plane of atoms over another plane of atoms. Stalder and Dürig have recently suggested that there should be a crossover from a dislocation-mediated slip to a homogeneous slip when the radius of a contact becomes smaller than a few nm. ${ }^{46}$ The reason for the crossover is that the energy of a dislocation in the middle of a cylinder of radius $R$ scales as $R \ln \left(R / r_{0}\right)$, whereas the energy required for a homogeneous slip scales as $R^{2}$. In this section, we investigate the slip mechanisms in detail using the nudged elastic band (NEB) method. ${ }^{47,48}$ The NEB method has been applied recently for modeling the formation of a contact between a Au tip and a Au surface. ${ }^{49}$

As an example, we consider the $\mathrm{Au}[110]$ contact. The 1st atomic rearrangement is a slip, which takes place in a single $\{111\}$ plane [Fig. 4(b)], and a stacking fault is introduced in the contact. If we compress the contact after the slip has occurred, there is a hysteresis effect: The contact keeps the structure containing the stacking fault beyond the point at which the slip occurred. For further compression, a "reverse" slip takes place at a contact length, $z_{2}$, which is 

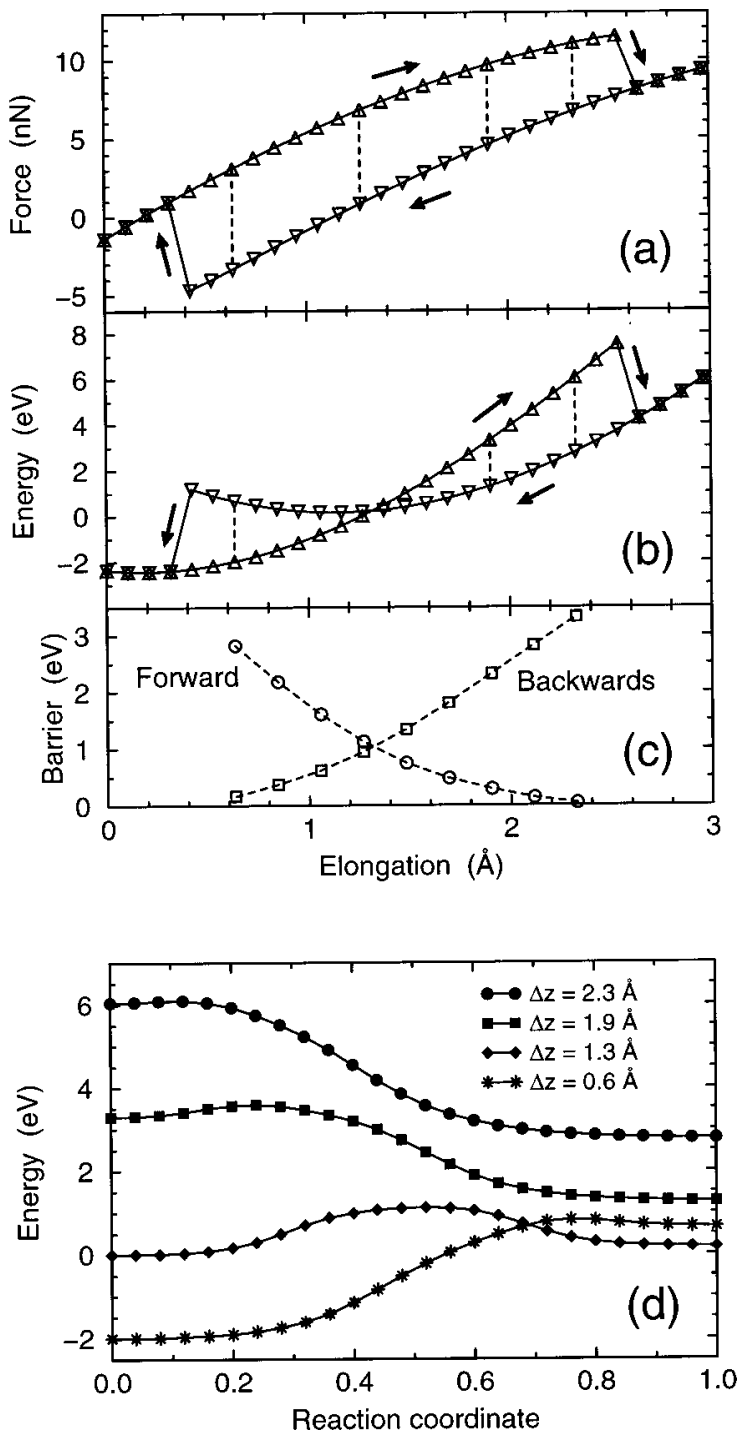

FIG. 11. A loading-unloading hysteresis loop for a slip process in $\mathrm{a} A[110]$ contact. (a) The tensile force and (b) potential energy during elongation and compression at $T=0 \mathrm{~K}$. (c) The potential energy barrier for the forward and backwards slip. (d) The potential energy along the MEP for four different contacts.

smaller than the length, $z_{1}$, at which the original slip occurred. By the reverse slip, the stacking fault is removed, and the original perfect structure is recovered. In Fig. 11(a) and Fig. 11(b) are shown the tensile force and potential energy, respectively, for an elongation-compression hysteresis loop at $T=0 \mathrm{~K}$.

The hysteresis reflects that for a contact with a length in the hysteresis range between $z_{2}$ and $z_{1}$, there exist two different (meta)stable atomic configurations: a "slipped" and an "unslipped" configuration, each corresponding to a local minimum of the potential energy. Initially, the unslipped system has the lowest energy, but as the contact is elongated, the slipped system becomes the most favorable. The reason for the hysteresis is that the two configurations are separated by an energy barrier (activation energy), and the contact will stay in a given configuration for a certain time, even when the other configuration has become more energetically favorable. At $T=0 \mathrm{~K}$, the transition from one configuration to the other occurs when the barrier vanishes. This happens when the energy minimum corresponding to the initial configuration vanishes, i.e., when the initial configuration becomes unstable. At a finite temperature, $T$, the transition occurs with a rate proportional to $\exp \left[-E_{\mathrm{a}} /\left(k_{\mathrm{B}} T\right)\right]$, where $E_{\mathrm{a}}$ is the energy barrier and $k_{\mathrm{B}}$ is the Boltzmann constant. Hysteresis effects in the loading-unloading behavior have been observed in experiments as well as in previous simulations. ${ }^{11,20}$ In general the situation is more complicated than sketched above, since there is not just one possible process but rather a variety of possible processes with different rates.

We have used the NEB method for calculating a minimum energy path (MEP) for the transition between the two metastable configurations for a number of different contact lengths [Fig. 11(d)]. The MEP is such that at any point along the path, the potential energy increases for displacements perpendicular to the path. In the NEB method, a discretized path of 26 replicas of the system is constructed by linear interpolation between the initial and final configurations, and then optimized iteratively. The energy barrier for a transition is given by the maximum energy along the MEP (at the transition state) minus the energy at the initial configuration. The energy barriers for the forward and backwards slips are shown in Fig. 11(c).

Our results offer an explanation why there is a good quantitative agreement between the MD simulations and experiments regarding the tensile strength, despite the large difference in time scales. At room temperature, a time scale difference of $10^{8}$ between MD simulations and experiments is equivalent to an increase in activation energy of about 0.5 $\mathrm{eV}$. But it can be seen from Fig. 11(c) that such a change in activation energy can be induced by a change in elongation on the order of $0.6 \AA$, which corresponds to a reduction in maximum force of only about $20 \%$. This estimate indicates that the increased time available for thermal activation in an experiment, compared to a MD simulation, does not reduce the tensile strength very much at room temperature or below for contacts of the present thickness.

The MEP obtained from the NEB calculation allows us to study in detail the motion of the atoms during a slip. The illustration of the transition state in Fig. 12(a) shows that the atoms at the slip plane move in an inhomogeneous manner. The slip occurs by glide of a Shockley partial dislocation nucleated at the surface of the contact. ${ }^{50}$ To investigate the question of size effects in the slip mechanisms, we have performed calculations for thinner $\mathrm{Au}[110]$ contacts with an otherwise similar shape, and the results support the notion of a size dependent crossover from dislocation glide to a homogeneous slip. For the contacts with diameters of $6.5 d_{\mathrm{NN}}$ or $5.5 d_{\mathrm{NN}}$, the slip mechanism is a dislocation glide, but for contacts with diameters of $4.5 d_{\mathrm{NN}}$ [see Fig. 12(b)] or $3.5 d_{\mathrm{NN}}$, the slip is homogeneous. The simulations suggest that the crossover diameter is around $15 \pm 3 \AA$.

\section{EFFECTS OF A SOFT CANTILEVER IN AN EXPERIMENT}

When comparing the simulation results to experimental measurements of force and conductance, caution is required in cases where the experimental setup has a small elastic stiffness. Rubio et al. have measured simultaneously the force and conductance during the formation and rupture of 


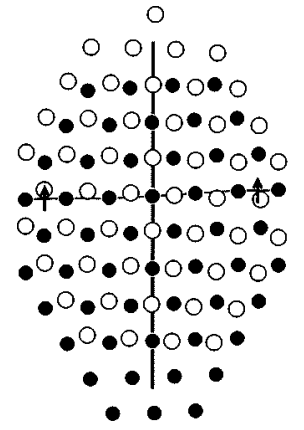

(a)

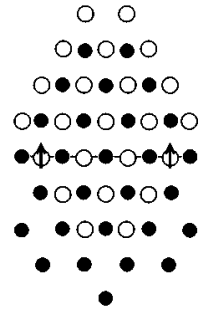

(b)
FIG. 12. The transition state for a slip in two $\mathrm{Au}[110]$ contacts. The atoms in the $\{111\}$ layers on each side of the slip plane are shown as white and black circles, respectively. The arrows show the displacement vector of the white circles. (a) A dislocation-mediated slip for a contact diameter of $6.5 d_{\mathrm{NN}}$. The thick line is a guide to the eye and it indicates an approximate location of the dislocation line. The arrows illustrate the Burgers vector of a Shockley partial dislocation, which moves from the left to the right in the picture. The dislocation is a screw dislocation since the Burgers vector is parallel to the dislocation line. (b) A homogeneous slip for a contact diameter of $4.5 d_{\mathrm{NN}}$.

atomic-sized $\mathrm{Au}$ contact using the following procedure. ${ }^{13} \mathrm{~A}$ sample of Au was mounted at the free end of a cantilever beam. A STM tip of Au was indented into the surface and then retracted. The indentation created a contact between the tip and sample, and during the tip retraction, the contact was stretched until it broke. The deflection of the cantilever was measured by an atomic force microscope (AFM), and the force was determined as the deflection of the cantilever multiplied by the effective spring constant of the cantilever. In order to get sufficiently large deflections for forces in the $\mathrm{nN}$ range, a soft cantilever was required. Rubio et al. used cantilevers with spring constants of $25 \mathrm{~N} / \mathrm{m}$ (Fig. 1 in Ref. 13) and $35 \mathrm{~N} / \mathrm{m}$ (Fig. 2 in Ref. 13). These values are on the order of the intrinsic effective spring constant of the nanocontact. In this section, we show that the results measured with a soft cantilever are significantly different from those that would have been obtained with a stiff setup.

In an experiment of the type described above, the length of the nanocontact cannot be directly controlled as opposed to the MD simulations. The inset in Fig. 13 shows a sketch of an (idealized) experimental setup. Instead of pulling the contact itself, one pulls a spring attached to the contact, and the mechanical response of the combined system consisting of the nanocontact and the apparatus (represented by the spring) is measured. The MD simulation results correspond to an infinitely large spring constant.

We model the influence of the cantilever as follows. The displacement of the upper end of the spring and the displacement of the nanocontact are denoted $z_{\mathrm{s}}$ and $z_{\mathrm{n}}$, respectively. Only the former can be directly controlled. From the MD simulations, we have calculated the tensile force as a function of the displacement of the nanocontact, $F\left(z_{\mathrm{n}}\right)$. Let us assume that we start the experiment with an unstretched contact and an unstretched spring. We now increase $z_{\mathrm{s}}$ stepwise, and in each step we determine a new (larger) value of $z_{\mathrm{n}}$ such that the system is balanced. In other words, for each value of $z_{\mathrm{s}}$, we increase (in small steps) $z_{\mathrm{n}}$ until the neck force $F\left(z_{\mathrm{n}}\right)$

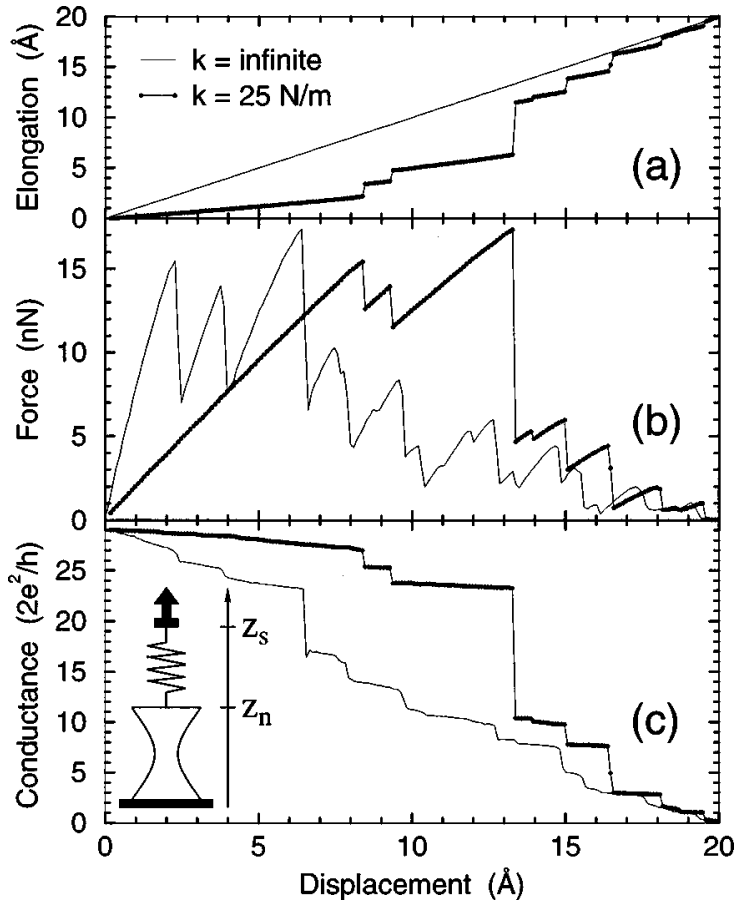

FIG. 13. The effect of a cantilever in an experiment. The thin solid lines show the MD simulation results from Fig. 2, and they correspond to an infinitely large effective spring constant of the cantilever. The circles are for a spring constant of $25 \mathrm{~N} / \mathrm{m}$ (same as in Fig. 1 in Ref. 13). (a) The elongation of the contact, $z_{\mathrm{n}}$, (b) the tensile force, and (c) the conductance are shown as a function of spring displacement, $z_{\mathrm{s}}$. Inset: Sketch of the model of the experiment.

equals the spring force $k \times\left(z_{\mathrm{s}}-z_{\mathrm{n}}\right)$. By this procedure, we can "transform" the simulated force curves and conductance curves, $F\left(z_{\mathrm{n}}\right)$ and $G\left(z_{\mathrm{n}}\right)$, into the experimental curves, $F\left(z_{\mathrm{s}}\right)$ and $G\left(z_{\mathrm{s}}\right)$.

Results of the model are shown in Fig. 13 for a spring constant of $k=25 \mathrm{~N} / \mathrm{m}$. The original simulation data are for the $\mathrm{Au}[111]$ nanocontact (Fig. 2). There are basically two effects of adding the spring to the system. First, in the elastic stages, the neck is stretched at a lower rate, because the spring is also stretched at the same time. Second, the springcontact system becomes unstable when the magnitude of the slope of the force curve (during the atomic rearrangements) exceeds the spring constant. This happens during the atomic rearrangements, and as a result, the contact is suddenly (discontinuously) stretched by a finite amount. Figure 13(a) shows how the length of the contact changes at a nonconstant rate, even though the end of the spring is pulled at a constant rate. It implies that certain ranges of $z_{\mathrm{n}}$ [and $F\left(z_{\mathrm{n}}\right)$ and $\left.G\left(z_{\mathrm{n}}\right)\right]$ are not probed.

The nonadiabatic deformation of the contact has very noticeable consequences for the measured force and conductance curves, as shown in Fig. 13(b) and Fig. 13(c). The slope of the force curve becomes smaller in the elastic stages. More importantly, when the length of the contact suddenly changes due to the instabilities in the springcontact system, intermediate peaks in the force curve may not be probed. Regarding the conductance curve, the plateaus appear more horizontal during the elastic deformation, and the conductance jumps by a larger amount during an 
instability, because a range of $G\left(z_{\mathrm{n}}\right)$ is skipped. Thus, a rather smooth conductance curve may transform into a stepped curve. The intermediate force peaks that are not detected, most often correspond to disordered contacts since these are generally weaker than well-ordered contacts. Another effect of the spring is that hysteresis effects in the loading-unloading behavior are exaggerated, since the spring is also elongated or compressed. Since information is lost in the experiment due to the instabilities, the measured results can not be "transformed" into the results that would have been obtained with a stiff cantilever.

\section{CONCLUSIONS}

In conclusion, we find a variety of different atomic rearrangement mechanisms dependent on the size, shape, crystal orientation, and degree of structural order of the contacts. For relatively thick contacts, the deformation preferentially takes place by slip in one or several close-packed $\{111\}$ planes. When slip occurs in several nonparallel planes, defects and disorder can be introduced. When disordered regions are present, the subsequent deformation mechanisms tend to involve the atoms in these regions, thereby changing the atomic structure and in general reducing the amount of disorder. In this way, disorder often anneals out again during the elongation process, but vacancies can be created and remain in the contacts. In this regime, the active zone extends over relatively many atomic layers in the contact. We observe a size-dependent crossover for the single-plane slip mechanism. For thicker contacts, the slip occurs by glide of a dislocation nucleated at the surface of the contact, whereas for thinner contacts, the slip is a homogeneous shear of one plane of atoms over another plane. The crossover takes place at a contact diameter around $15 \pm 3 \AA$.

When the wire has become relatively thin, the nature of the deformation changes. The atomic rearrangements become more irregular and localized; the active zone typically extends over 2-4 layers. Contacts with an oblong cross section, perpendicular to the $z$ direction, tend to become perma- nently disordered in the narrowest region for the last part of the elongation until rupture of the contact. This mainly happens for contacts oriented along the [110] or the [100] direction, whereas contacts oriented along the [111] direction are more rotationally symmetric. Disordered contacts tend to become longer and thinner than ordered contacts in the last part of the elongation. We find that contacts with regions of structural disorder are weaker than well-ordered contacts of similar thickness.

We have shown that caution is required when comparing results of computer simulations to experimental results obtained with a setup that has a stiffness comparable to the stiffness of the nanocontact. A small stiffness can, for instance, arise from a soft cantilever or from the flexibility of macroscopic wires. As an effect of a soft apparatus, the length of the nanocontact cannot be controlled directly. Due to instabilities in the cantilever-contact system, there are contact lengths that are not probed during an experiment, and as a consequence, one may not detect all atomic rearrangements. Conductance plateaus become more flat, and jumps in conductance or force become more pronounced. Wellordered contact structures, which are relatively strong, will tend to be probed rather than weaker, disordered structures.

\section{ACKNOWLEDGMENTS}

We thank Urs Dürig for suggesting to us the importance of a soft cantilever in experimental force measurements. We are indebted to Per Stoltze, Jakob Schiøtz, and S. G. Srinivasan for using their respective computer code, and we thank Hannes Jónsson for many valuable discussions, in particular on the NEB method. Helpful and stimulating discussions with Erik Lægsgaard, Kim Hansen, Lars Olesen, Flemming Besenbacher, Jens K. Nørskov, and Francesco di Tolla are gratefully acknowledged. CAMP, the Center for Atomicscale Materials Physics, is sponsored by the Danish National Research Foundation. Further funding has been obtained from the Danish Research Councils through the Center for Nano-Tribology and from the U.S. Department of Energy through Grant No. DEFG02-88ER45364.
${ }^{1}$ G. Binnig, H. Rohrer, Ch. Gerber, and E. Weibel, Phys. Rev. Lett. 49, 57 (1982).

${ }^{2}$ C. J. Muller, J. M. van Ruitenbeek, and L. J. de Jongh, Phys. Rev. Lett. 69, 140 (1992).

${ }^{3}$ N. Agraï, J. G. Rodrigo, and S. Vieira, Phys. Rev. B 47, 12345 (1993).

${ }^{4}$ J. I. Pascual, J. Méndez, J. Gómez-Herrero, A. M. Baró, N. García, and V. T. Binh, Phys. Rev. Lett. 71, 1852 (1993).

${ }^{5}$ N. Agraï, J. G. Rodrigo, C. Sirvent, and S. Vieira, Phys. Rev. B 48, 8499 (1993).

${ }^{6}$ L. Olesen, E. Lægsgaard, I. Stensgaard, F. Besenbacher, J. Schiøtz, P. Stoltze, K. W. Jacobsen, and J. K. Nørskov, Phys. Rev. Lett. 72, 2251 (1994).

${ }^{7}$ J. I. Pascual, J. Méndez, J. Gómez-Herrero, A. M. Baró, N. García, U. Landman, W. D. Luedtke, E. N. Bogachek, and H.-P. Cheng, Science 267, 1793 (1995).

${ }^{8}$ V. V. Dremov, S. Yu. Shapoval, and E. V. Sukhorukov, Phys. Low-Dimens. Semicond. Struct. 11/12, 29 (1994).
${ }^{9}$ M. Brandbyge, J. Schiøtz, M. R. Sørensen, P. Stoltze, K. W. Jacobsen, J. K. Nørskov, L. Olesen, E. Lægsgaard, I. Stensgaard, and F. Besenbacher, Phys. Rev. B 52, 8499 (1995).

${ }^{10}$ Z. Gai, Y. He, H. Yu, and W. S. Yang, Phys. Rev. B 53, 1042 (1996).

${ }^{11}$ N. Agraï, G. Rubio, and S. Vieira, Phys. Rev. Lett. 74, 3995 (1995).

${ }^{12}$ A. Stalder and U. Dürig, J. Vac. Sci. Technol. B 14, 1259 (1996).

${ }^{13}$ G. Rubio, N. Agrait, and S. Vieira, Phys. Rev. Lett. 76, 2302 (1996).

${ }^{14}$ J. M. Krans, C. J. Muller, I. K. Yanson, Th. C. M. Govaert, R. Hesper, and J. M. Van Ruitenbeek, Phys. Rev. B 48, 14721 (1993).

${ }^{15}$ J. M. Krans, J. M. van Ruitenbeek, V. V. Fisun, I. K. Yanson, and L. J. de Jongh, Nature (London) 375, 767 (1995).

${ }^{16}$ C. J. Muller, J. M. Krans, T. N. Todorov, and M. A. Reed, Phys. Rev. B 53, 1022 (1996).

${ }^{17}$ J. L. Costa-Krämer, N. García, P. García-Mochales, and P. A. 
Serena, Surf. Sci. 342, L1144 (1995); 349, L138 (1996).

${ }^{18}$ J. L. Costa-Krämer, N. García, P. García-Mochales, P. A. Serena, M. I. Marqués, and A. Correia, Phys. Rev. B 55, 5416 (1997).

${ }^{19}$ J. L. Costa-Krämer, Phys. Rev. B 55, R4875 (1997).

${ }^{20}$ U. Landman, W. D. Luedtke, B. E. Salisbury, and R. L. Whetten, Phys. Rev. Lett. 77, 1362 (1996).

${ }^{21}$ K. Hansen, E. Lægsgaard, I. Stensgaard, and F. Besenbacher, Phys. Rev. B 56, 2208 (1997).

${ }^{22}$ U. Landman, W. D. Luedtke, N. A. Burnham, and R. J. Colton, Science 248, 454 (1990).

${ }^{23}$ A. P. Sutton, J. B. Pethica, H. Rafii-Tabar, and J. A. Nieminen, in Electron Theory in Alloy Design, edited by D. G. Pettifor and A. H. Cottrell (Institute of Materials, London, 1992), Chap. 7.

${ }^{24}$ T. N. Todorov and A. P. Sutton, Phys. Rev. Lett. 70, 2138 (1993).

${ }^{25}$ A. M. Bratkovsky, A. P. Sutton, and T. N. Todorov, Phys. Rev. B 52, 5036 (1995).

${ }^{26}$ T. N. Todorov and A. P. Sutton, Phys. Rev. B 54, R14 234 (1996).

${ }^{27}$ J. A. Torres and J. J. Sáenz, Phys. Rev. Lett. 77, 2245 (1996).

${ }^{28}$ M. P. Allan and D. J. Tildesley, Computer Simulations of Liquids (Clarendon, Oxford, 1987), pp. 259-263.

${ }^{29}$ P. Stoltze, Simulation Methods in Atomic-Scale Materials Physics (Polyteknisk Forlag, Lyngby, 1997), pp. 77-78.

${ }^{30}$ The value of the friction coefficient in the Langevin dynamics, $\xi=0.001 \times(\Delta t)^{-1}$, has been chosen from a compromise: on the one hand, it should be as small as possible in order not to disturb the dynamics of the system more than necessary. On the other hand, it must be high enough to allow the increase in kinetic energy during an atomic rearrangement to be dissipated before the next rearrangement takes place.

${ }^{31}$ K. W. Jacobsen, J. K. Nørskov, and M. J. Puska, Phys. Rev. B 35, 7423 (1987).

${ }^{32}$ P. Stoltze, J. Phys.: Condens. Matter 6, 9495 (1994).

${ }^{33}$ K. W. Jacobsen, P. Stoltze, and J. K. Nørskov, Surf. Sci. 366, 394 (1996).

${ }^{34}$ A. S. Clarke and H. Jónsson, Phys. Rev. E 47, 3975 (1993).

${ }^{35}$ D. Faken and H. Jónsson, Comput. Mater. Sci. 2, 279 (1994).

${ }^{36}$ H. Jónsson and H. C. Andersen, Phys. Rev. Lett. 60, 2295 (1988).

${ }^{37}$ Besides the types of pairs mentioned above, there is also a significant number of pairs of type 322 and 300 present, but we have found that they do not reveal any additional useful information. The remaining types of pairs are relatively rare. We note in particular that pairs that are characteristic of bcc or simple cubic ordering are extremely rare.

${ }^{38}$ The most important factor is the potential near the narrowest part of the contact; the conductance does not depend in any essential way on the particular way the joining is made. Only the "active" middle part of the contact is used in the conductance calculation. For instance, for the $\mathrm{Au}[111]$ contact (Fig. 1) the freeelectron electrodes are attached to the contact at a distance of six atomic layers from the top and bottom, respectively.

${ }^{39}$ H. L. Skriver and N. M. Rosengaard, Phys. Rev. B 46, 7157 (1992).

${ }^{40}$ R. Landauer, IBM J. Res. Dev. 1, 223 (1957).

${ }^{41}$ M. Büttiker, Y. Imry, R. Landauer, and S. Pinhas, Phys. Rev. B 31, 6207 (1985).

${ }^{42}$ M. Brandbyge, K. W. Jacobsen, and J. K. Nbrskov, Phys. Rev. B 55, 2637 (1997).

${ }^{43}$ The fluctuations in the number of pairs are in themselves indicators of an unusual atomic environment. The fluctuations can be caused by atoms moving in and out of the cutoff range for neighbor atoms. This happens when there are subpeaks in the radial distribution function (RDF) at the cutoff distance. For a contact with a perfect fcc structure, there are no such peaks, since the cutoff distance is chosen to be exactly in between the positions of the first and second peaks of the RDF in fcc bulk.

${ }^{44}$ B. Hammer and J. K. Nørskov, Surf. Sci. 343, 211 (1995), and references therein.

${ }^{45}$ L. Olesen, Ph.D. thesis, University of Aarhus, 1996.

${ }^{46}$ A. Stalder and U. Dürig (unpublished).

${ }^{47}$ M. Villarba and H. Jónsson, Surf. Sci. 317, 15 (1994).

${ }^{48}$ G. Mills, H. Jónsson, and G. K. Schenter, Surf. Sci. 324, 305 (1995).

${ }^{49}$ M. R. Sørensen, K. W. Jacobsen, and H. Jónsson, Phys. Rev. Lett. 77, 5067 (1996).

${ }^{50}$ This kind of dislocation nucleation and propagation has also been observed for sliding friction between a $\mathrm{Cu}$ tip and a $\mathrm{Cu}$ surface in M. R. Sørensen, K. W. Jacobsen, and P. Stoltze, Phys. Rev. B 53, 2101 (1996). 